\title{
Artificial neural networks as the data ruling components of future intelligent energy systems
}

\author{
Anna Buran ${ }^{1, *}$, and Maksim Denisov ${ }^{1}$ \\ ${ }^{1}$ National Research Tomsk Polytechnic University, 634050 Tomsk, Russia
}

\begin{abstract}
The main target of this work is to study artificial neural networks and their role in the future intelligent energy systems. Artificial neural networks in the near future will be a very effective tool for data analysis and very perspective for energy consumers. The accelerating growth in the field of software engineering research will prove appearing of neural networks in intelligent energy systems. This study is expected to be important for researchers and engineers studying artificial neural networks (ANNs) in a wide range of domains.
\end{abstract}

\section{Introduction}

Researchers and scientists study artificial neural networks (ANNs) in different spheres of our life where neural networks can be used, from economics, business, computer games to induction motors. The artificial neural network was designed as a computational model based on the brain to solve particular problems. In the modern world ANNs take the form of different calculating, data-analyzing, computing algorithms which can already be used in our life, for example, by any mobile phone owner who desires for fun to change his appearance in the photo - it can be provided by such mobile apps as MSQRD and Prisma, based on neural algorithms. Some scientists try to predict the accuracy of components produced by Wire Electrical Discharge Machining (WEMD) by using an Elman-based Layer Recurrent Neural Network (LRNN) [1]. Several studies discuss a novel conceptual framework: Fractional Hopfield neural networks (FHNN), neural network-based command filtered control for induction motors with input saturation, design of a neural network based distributed power flow controller (DPFC) for power system stability, while others observe the feedback principle $[2,3,4,5]$. Deisseroth and Schnitzer study engineering approaches to illuminating brain structure and dynamics. Electrical engineering has long influenced neuroscience, having contributions in cable theory [6, 7] and radioelectronics [7]. This study is aimed at investigating the role of future intellectual energy systems in the field of neuronal creation. First, the principles on which neuronets are based should be considered.

\footnotetext{
* Corresponding author: aburan@tpu.ru
} 


\subsection{Neurons, synaptic connections, synapse weight, activation functions}

We can suggest that neural networks are inspired by the biological neural networks of animal and, correspondingly, human brains. Heaton studied the influence of artificial intelligence for humans [8]. One of the main advantages of these algorithms - progressive self learning process based on considering examples and learning from mistakes; the same, but much more complicated logics is developed by nature in human brains. When we refer to artificial neural networks, we deal with the following definitions which came from biology. 1) Neuron - in ANN is a mathematical function, or computing unit which receives information (in the form of signal), calculates it and transmits further. There are three main types of neurons: input, covered and output. Input neurons usually sum signals of another quantity of neurons and using activation functions output information; covered work with the information received from other covered or input neurons; output neurons give the final answer to indignation of this information system.

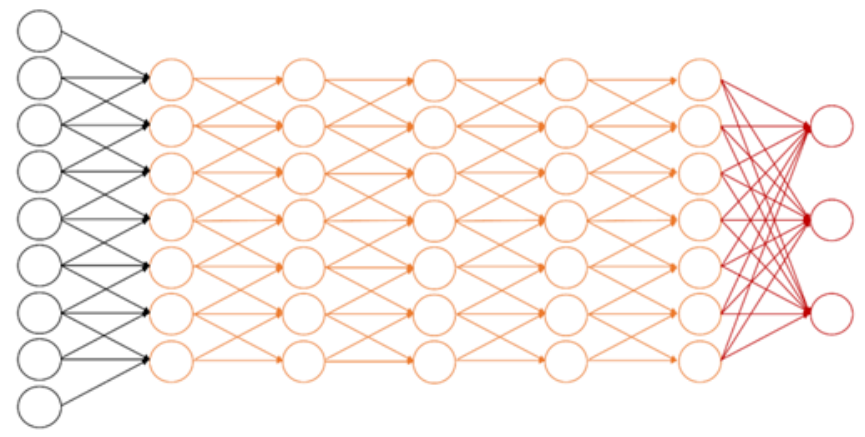

Fig. 1. Different types of neurons.

Synaptic connection exists between two neurons. Synapses have one important parameter - synapses weight. It's responsible for processing the information, and on the whole all the synaptic weights of ANNs composes "the brain" of the system. The synapse weight can be demonstrated with the help of colour mix (fig. 1) - mixing colours in the right proportion (due to their "weight" in new composed colour) creates a new colour.

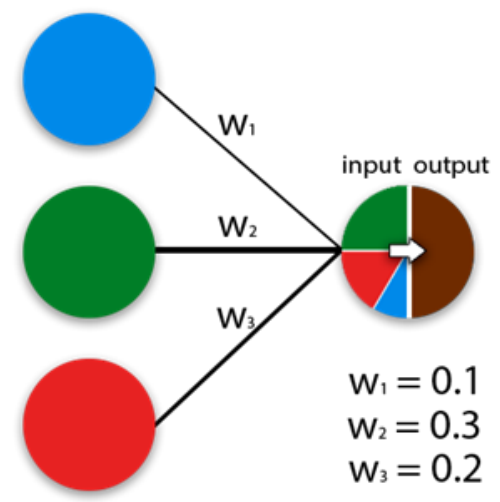

Fig. 2. Synapses weight demonstration on the mix of colours. W - weight.

The following figure shows input and output data producing, where I1, I2 are input neurons, $\mathrm{H} 1$ is covered (output) neuron, w1, w2 are synaptic weights. 


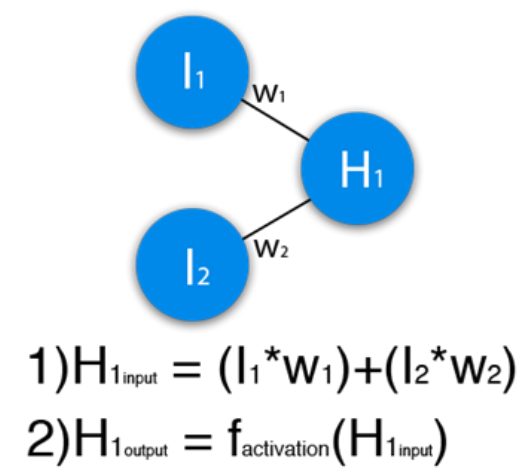

Fig. 3. Input and output data producing. I1, I2 - input neurons, H1 - covered (output) neuron, w1, w2 - synaptic weights.

Output information is a function of neuron activation. Activation function is the function of data normalization, in other words - how the quantity of signals which come to income neurons turn into one signal. Once the ANN's ready for activation, it begins repeating data analysis. After several attempts to calculate/compute right answer, it makes mistakes which are factored into the next calculating attempt. If the system calculates the right answer, it will always be able to work with this type of work without any mistake.

\subsection{ANN in intellectual energy systems}

Artificial neural networks in smart grids can input data, analyzing programs effectively and responding the needs of certain energy consumers.

What are the characteristics of the future smart grids which require ANN abilities?

1) Flexibility of different energy source usage, including all types of fossil fuels and renewable sources allowable for certain energy consumer;

2) Stability in the energy system of consumer which requires "smart" decisions close in the compartment with decisions made by human;

3) Increasing of energy efficiency connected directly with the information about the energy source supply, which in turn is connected with global net information (as, for example, the weather forecast connected with the energy supply of a certain detached house).

What sources of energy and information will be the input information for ANN?

1) ANN will control the quantity of allowable renewable sources - wind, solar, geothermal energy which is not constant during, for example, one period of usage (day, month, year); 2) ANN will control the usage of the main grid sources - electricity got from electrical grid of country region;

3) Information directly connected with the renewable source generation (like weather forecast) will be getting from Web sources, so ANN will build energy consumption plans for the day/month.

But if we consider ANNs, we have to answer the next question: which way is ANN going to learn how to rule allowed resources and how to manage information about their existence. There are several foreseeable decisions to this problem.

1.ANN's actions before its utilization in real energy systems are going to be "trained" in special conditions by experienced people on the base of computer centers. Training will be similar to the training of modern ANN's with its repeated question-answer-mistake circle. Then trained ANN will be implemented into a real energy system with its conditions similar to conditions ANN learnt in. 
2.ANN's background will be fully built in special computer centers, but the real studying of ANN has already occurred in the consumer's energy system. The studying will be provided by active dialogue with consumers on the base of human interface connection with question-answer logics.

\section{Conclusion}

Artificial neural networks in the foreseeable future will be able to become a very effective tool for data analysis, for self studying and very perspective for smart grids of energy consumers from detached houses to little industries. The accelerating growth in the field of software engineering research will prove appearing of ANNs very soon.

\section{References}

1. A. Conde, A. Arriandiaga, J. A.Sanchez, E. Portillo, S. Plaza, I. Cabanes, Robot Comput Integr Manuf. (to be published)

2. Y.-F. Pu, Z. Yi, J.-L. Zhou, IEEE Trans. Neural Netw. Learn. Syst. 28 (2017)

3. C. Fu, L. Zhao, J. Yu, H. Yu, C. Lin, IET Control Theory Appl. 11 (2017)

4. G.M. Rao, V.A. Kumar, B.V.S. Ram, International Conference on Signal Processing, Communication, Power and Embedded System, SCOPES 2016 - Proceedings 7955509 (2017)

5. A. L. Buran, V.E. Kozlov, A.V. Filyukov, MATEC Web Conf. 110, 01021 (2017)

6. T. V. Susakova, A.M. Evseeva, I.A. Chesnokova, MATEC Web Conf., 110, 01077 (2017)

7. K. Deisseroth, M. J. Schnitzer, NEURON, 80 (3) (2013)

8. J. Heaton, Artificial Intelligence for Humans. Volume 1: Fundamental Algorithms (CreateSpace Independent Publishing, North Charleston, 2013) 